\title{
Reduction of atlantoaxial dislocation prevented by pathological position of the transverse ligament in fixed, irreducible os odontoideum: operative illustrations and radiographic correlates in $\mathbf{4 1}$ patients
}

\author{
Brian J. Dlouhy, MD, ${ }^{1}$ Bruno A. Policeni, MD, ${ }^{2}$ and Arnold H. Menezes, MD' \\ Departments of ${ }^{1}$ Neurosurgery and ${ }^{2}$ Radiology, University of lowa Hospitals and Clinics, lowa City, lowa
}

OBJECTIVE Os odontoideum (OO) is a craniovertebral junction (CVJ) abnormality in which an ossicle (small bone) is cranial to a hypoplastic dens by a variable gap. This abnormality can result in instability, which may be reducible or irreducible. What leads to irreducibility in $\mathrm{OO}$ is unclear. Therefore, the authors sought to better understand the causes of irreducibility in 00.

METHODS A retrospective review was conducted, which identified more than 200 patients who had undergone surgical treatment for $\mathrm{OO}$ between 1978 and 2015 at the University of lowa Hospitals and Clinics. Only the 41 patients who had irreducible $\mathrm{OO}$ were included in this study. All inpatient and outpatient records were retrospectively reviewed, and patient demographics, clinical presentation, radiographic findings, surgical treatment, and operative findings were recorded and analyzed.

RESULTS The cohort of 41 patients who were found to have irreducible 00 included both children and adults. A majority of patients were adults (61\% were 18 years or older). Clinical presentation included neck pain and headache in the majority of patients (93\%). Weakness, sensory disturbances, and myelopathy were invariably present in all 41 patients $(100 \%)$. Down syndrome was much more common in the pediatric cohort than in the adult cohort; of the 16 pediatric patients, 6 had Down syndrome (38\%), and none of the adults did. Of the 16 pediatric patients, 5 had segmentation failure $(31 \%)$ in the subaxial spine, and none of the adults did. A form of atlantoaxial dislocation was seen in all cases. On CT imaging, atlantoaxial facets were dislocated in all 41 cases but did not have osseous changes that would have prevented reduction. On MRI, the transverse ligament was identified anterior and inferior to the ossicle and superior to the hypoplastic odontoid process in all cases in which these studies were available (i.e., post-MRI era; 36 of 36 cases). The ligament was hypointense on T2-weighted images but also had an associated hyperintense signal on T2 images. Intraoperatively, the transverse ligament was identified anterior and inferior to the ossicle and superior to the hypoplastic odontoid process in all 41 cases.

CONCLUSIONS In the largest series to date of irreducible $\mathrm{OO}$ and the only study to examine variable factors that lead to irreducibility in $\mathrm{OO}$, the authors found that the position of the transverse ligament anterior and inferior to the ossicle is the most common factor in the irreducibility of $\mathrm{OO}$. The presence of granulation tissue and of the dystopic variant of 00 is also associated with irreducibility. The presence of Down syndrome and segmentation failure probably leads to faster progression of ligamentous incompetence and therefore earlier presentation of instability and irreducibility. This is the first study in which intraoperative findings regarding the transverse ligament have been correlated with MRI.

https://thejns.org/doi/abs/10.3171/2016.11.SPINE16733

KEY WORDS transoral approach; cruciate ligament; transverse ligament; os odontoideum; reducible; irreducible; craniovertebral junction; craniocervical junction; congenital 
$\mathrm{O}$ s odontoideum $(\mathrm{OO})$ is a rare craniovertebral junction (CVJ) abnormality in which an ossicle (small bone) of varying size and shape is separated from a hypoplastic odontoid process (OP) by a variable gap. ${ }^{8,12}$ The ossicle is cranial to the hypoplastic dens, has rounded and smooth cortical borders, and has no osseous continuity with the hypoplastic dens or the body of C-2.8

As with all CVJ abnormalities, $\mathrm{OO}$ has been categorized based on its instability and reducibility. ${ }^{2,5,6,12}$ Therefore, a proper assessment of all patients with $\mathrm{OO}$ requires dynamic CVJ studies-CVJ flexion-extension MRI-to determine the presence of instability and whether this instability, if present, is reducible or irreducible.$^{5,13-15} \mathrm{Re}$ ducibility pertains to the reestablishment of anatomical alignment to relieve compression of neural structures. ${ }^{5}$ Instability requires treatment, and whether $\mathrm{OO}$ is reducible dictates proper treatment strategy. If $\mathrm{OO}$ is irreducible with flexion or extension, skeletal traction, intraoperative $\mathrm{O}$-arm reduction, or posterior intraoperative distraction and reduction, a ventral decompression is required prior to posterior fusion. ${ }^{5,12,22}$

Because the gap between the ossicle and the body of C-2 usually extends above the level of the superior facets of $\mathrm{C}-2$, over time this leads to incompetence of the transverse cruciate ligament and subsequently, C1-2 (atlantoaxial) instability and dislocation. ${ }^{6,12}$ However, why some cases of $\mathrm{OO}$ develop a fixed dislocation and therefore become irreducible and others do not is unclear and has not been investigated. Therefore, to assess the cause of this irreducibility and better understand the pathophysiology of $\mathrm{OO}$, we retrospectively examined all cases of irreducible OO treated at the University of Iowa Hospitals and Clinics between 1978 and 2015.

\section{Methods}

\section{Patient Population}

A retrospective review identified more than 200 patients who underwent surgical treatment by the senior author (A.H.M.) for OO between 1978 and 2015 at the University of Iowa Hospitals and Clinics. Irreducible OO occurred in 41 patients. All inpatient and outpatient records were retrospectively reviewed, and the following data and variables were recorded: patient age, sex, clinical presentation and examination findings, anatomical classification of $\mathrm{OO}$, presence of atlantoaxial dislocation and instability, associated Chiari I malformation, associated basilar invagination, associated atlas assimilation, surgical management, and associated operative findings. This study was approved by the University of Iowa Institutional Review Board.

\section{Management of $\mathrm{OO}$ at the University of lowa}

The University of Iowa approach to abnormalities at the CVJ has been described in detail and extensively throughout the literature. ${ }^{5,15}$ Since 1978, the approach and management of CVJ abnormalities has evolved significantly but the basic principles have remained the same. ${ }^{5}$ Prior to 1984, all patients underwent plain cervical radiography and pluridirectional tomography in the flexed, extended, and lateral bending positions. This was sup- plemented with CT imaging, CT myelography, vertebral angiography when necessary, and documentation of the effects of cervical traction. After 1984, CT myelography was replaced with MRI, and vertebral angiography was replaced with CT angiography. Today, a CVJ cervical CT and flexion-extension MRI are obtained in all patients, along with CT angiography if needed. Furthermore, 3D $\mathrm{CT}$ imaging of the CVJ has been performed in all patients since 1990. This imaging allows assessment of osseoligamentous abnormalities, determines instability, assesses reducibility, and determines the optimum position for fusion if needed. Specific to OO, all patients with instability are treated with surgical management. Unstable but reducible $\mathrm{OO}$ requires an occipitocervical or atlantoaxial fusion, depending on the levels of instability. Irreducible $\mathrm{OO}$ is treated with intraoperative $\mathrm{O}$-arm reduction. If this fails, a transoral approach is used to decompress the cervicomedullary junction (CMJ) anteriorly. After the transoral decompression, a posterior occipitocervical fusion is performed.

\section{Results}

\section{Patient Demographics, Clinical Presentation, and Examination Findings}

Both children and adults presented with irreducible OO (Table 1). The average age at the time of surgery was 26 years (range 5-52 years). A majority of patients were adults; 16 patients (39\%) were younger than 18 years (pediatric), and 25 (61\%) were 18 years or older (adult). Down syndrome was more common in the pediatric cohort than in the adult cohort. Of the 16 pediatric patients, 6 had Down syndrome (38\%), whereas of the 25 adult patients, none did. A total of 19 male and 22 female patients underwent preoperative evaluation and surgery. Clinical presentation included neck pain and headache in the majority of patients (93\%). Weakness, sensory disturbances, and myelopathy were invariably present in all 41 patients $(100 \%)$.

\section{Radiographic Findings}

Using the anatomical classification for $\mathrm{OO}, 40 \mathrm{pa}-$ tients (98\%) were found to have a dystopic OO, and only 1 patient $(2 \%)$ had an orthotopic OO (Table 1). All cases resulted in some form of atlantoaxial dislocation and instability (100\%). Associated Chiari I malformation was seen in 3 patients (7\%). Basilar invagination and atlas assimilation were each seen in 6 patients $(15 \%)$. Associated segmentation failure in the subaxial spine was much more common in the pediatric cohort than in the adult cohort. Of the 16 pediatric patients, 5 had segmentation failure (31\%) in the subaxial spine, whereas of the 25 adult patients, none had segmentation failure. A form of atlantoaxial dislocation was seen in all 41 cases. On MRI, the transverse ligament was identified anterior and inferior to the ossicle and superior to the hypoplastic OP in all cases in which these studies were available (i.e., post-MRI era; 36 of 36 cases) (Fig. 1). The ligament was hypointense but also had an associated hyperintense signal on T2-weighted MRI, probably from edematous changes. On CT imaging, in all 41 cases, atlantoaxial facets were dislocated but 
TABLE 1. Demographics, clinical presentation, radiographic findings, and operative findings in 41 patients with irreducible 00

\begin{tabular}{lc}
\hline \multicolumn{1}{c}{ Characteristic } & Value (\%) \\
\hline Mean age in yrs, range & $26,5-52$ \\
\hline Male & $19(46)$ \\
\hline Clinical presentation \& examination findings & $38(93)$ \\
\hline Neck pain \& headache & $41(100)$ \\
\hline Weakness & $41(100)$ \\
\hline Sensory disturbances & $41(100)$ \\
\hline Myelopathy & \\
\hline Anatomical classification of OO & $40(98)$ \\
\hline Dystopic & $1(2)$ \\
\hline Orthotopic & $41(100)$ \\
\hline Radiographic findings & $41(100)$ \\
\hline Atlantoaxial dislocation \& instability & $0(0)$ \\
\hline Irreducible & $3(7)$ \\
\hline Osseous changes to C1-2 facets that prevent & $6(15)$ \\
\hline reduction & $6(15)$ \\
\hline Chiari I malformation & $36 / 36(100)$ \\
\hline Basilar invagination & \\
\hline Atlas assimilation & $19(46)$ \\
\hline Transverse ligament inferior \& anterior to ossicle \\
on MRI
\end{tabular}

did not have osseous changes that would have prevented reduction (Fig. 2).

\section{Operative Findings}

All 41 patients with irreducible OO underwent a transoral decompressive surgery (Table 1, Video 1).

VIDEO 1. Clip showing transoral approach for irreducible 00 , with illustration of the pathological position of the transverse ligament preventing reduction (Case 2). This video is an operative illustration of the pathological position of the transverse ligament and its radiographic correlate in a 27 -year-old man with irreducible 00. This video highlights and provides a better spatial orientation of the transverse ligament to the $\mathrm{OO}$ in these irreducible cases. Copyright Brian J. Dlouhy. Published with permission. Click here to view.

In all patients, the $\mathrm{C}-1$ anterior tubercle and $\mathrm{C}-1$ anterior arch was resected to expose the OO. In 13 cases the $\mathrm{OO}$ had migrated significantly upward into the foramen magnum, necessitating clival removal. Intraoperatively, the transverse ligament was identified anterior and inferior to the ossicle and superior to the hypoplastic OP in all 41 cases (Figs. 3 and 4). The transverse ligament projected slightly superior and anterior, which was quite different than the typical position behind the odontoid seen in other pathologies that have required a transoral decompressive procedure. This pathological position of the transverse ligament required us to work behind the ligament to first core out and then curette out the OO. The ossicle was fixed behind the ligament, preventing reduction. Intense granulation tissue adjacent to the ossicle was present in 38 patients.

\section{Illustrative Cases \\ Case 1}

This 27-year-old man presented with a 9-month history of midcervical neck pain and a 4-week history of numbness and paresthesias on the left side of his body. These symptoms worsened with lying down and neck flexion. He also reported an abnormal clicking sensation in his neck and was able to alleviate this with neck extension, twisting, and manipulation. On physical examination, he had good neck motion. Strength was maintained throughout $(4+/ 5)$. Deep tendon reflexes were hyperreflexic $(3 / 4+)$ throughout in the bilateral upper and lower extremities. He had bilateral Hoffman and Babinski responses. He had ankle clonus and patella clonus bilaterally.

Cervical spine radiographs with flexion-extension showed a dystopic OO with atlantoaxial dislocation (Fig. 1). In flexion the axis dislocated from the atlas, causing severe canal compromise by the axis body. The patient himself was able to manipulate his neck, and with extension he was able to reduce this dislocation. This was confirmed with cervical spine flexion-extension radiographs. He was then referred to us. A flexion-extension MRI sequence revealed atlantoaxial dislocation, severe spinal canal stenosis, and cord signal change at the CMJ on T2-weighted imaging. However, at the time this MRI was done, reduction was not observed with extension. The transverse ligament was observed inferior and anterior to the ossicle and superior to the hypoplastic dens and C-2 body. Crown halo cervical traction was applied and traction weight was gradually increased from 10 to $16 \mathrm{lbs}$, but reduction and correction of alignment was not achievable by this point.

The patient was maintained in crown halo cervical traction and underwent a transoral-transpalatopharyngeal approach to the CVJ. The C-1 anterior tubercle and C-1 anterior arch were resected. We then recognized that the transverse ligament was superior to the hypoplastic dens and C-2 body, and that the $\mathrm{OO}$ was posterior to the transverse ligament. The ossicle could not be moved forward because of the transverse ligament, and hence the ossicle was removed as well as the superior aspect of the hypoplastic OP. A dorsal Oc-C3 instrumentation with fusion with rib graft was subsequently performed. Intraoperatively, his reduction was satisfactory. He made a complete neurological recovery.

\section{Case 2}

This 27-year-old man reported severe neck pain and dizziness that had lasted for 2 months; both worsened with his job as a construction worker. With exertion, he became short of breath and experienced weakness in his hands and paresthesias in his hands and legs. He had a history of trauma as a 3-year-old and his mother had been told that 

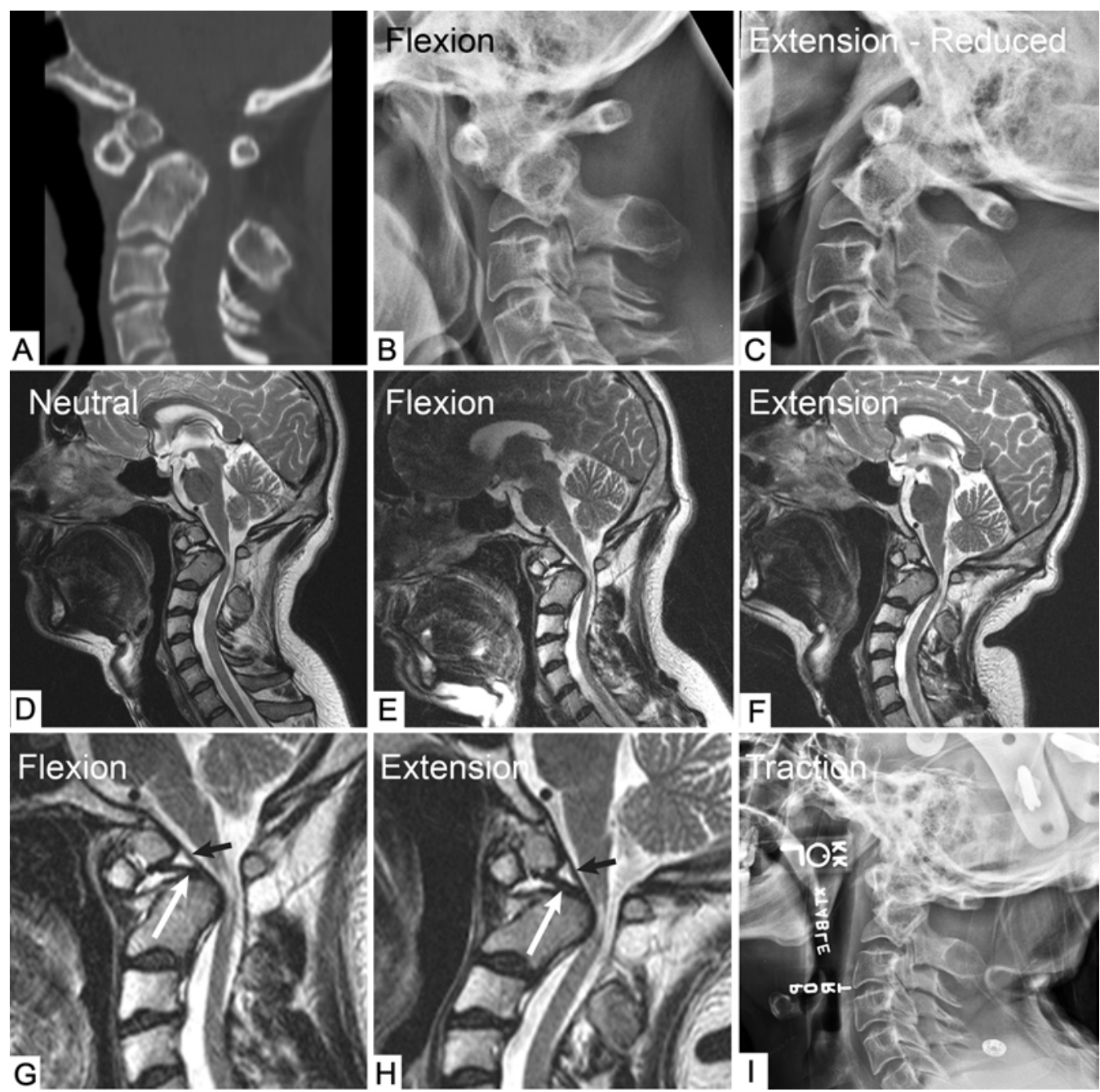

FIG. 1. Case 1. A 27-year-old man initially presented with a reducible $\mathrm{OO}$ and atlantoaxial dislocation-however the $\mathrm{OO}$ became irreducible over time, due to pathological positioning of the transverse ligament. Preoperative sagittal CT (A) revealing a dystopic $\mathrm{OO}$ and atlantoaxial dislocation. Flexion (B) and extension (C) cervical spine radiographs initially demonstrated atlantoaxial reducibility and proper $\mathrm{Oc}-\mathrm{C} 2$ alignment with neck manipulation and extension. Ultimately, this became irreducible and the patient was unable to extend and manipulate his neck to achieve reducibility (D); this is clearly seen in flexion (E) and extension (F) MRI. Flexion (G) and extension (H) sagittal T2-weighted MRI revealing irreducible $\mathrm{OO}$ and atlantoaxial dislocation and severe cervicomedullary compression and T2 cord signal change. The transverse ligament (white arrows) is hypointense with an associated hyperintense signal, probably from edematous changes. The transverse ligament is anterior and inferior to the ossicle, and has thus the ability to assist in reduction with head position. The tectorial membrane (black arrows) lies in its normal position. Even in traction (I), the $\mathrm{OO}$ and atlantoaxial dislocation was irreducible. The patient subsequently underwent transoral decompression confirming the radiographic findings regarding the position of the transverse ligament.

he was born with an upper cervical spine abnormality. On physical examination, the main findings were that he had weakness in his hands and feet and was grossly ataxic. $\mathrm{He}$ was hyperreflexic throughout his body.

Imaging of the CVJ revealed a dystopic $\mathrm{OO}$ with compression of the ventral CMJ (Fig. 3). There was hourglass constriction of the CMJ with hyperintense cord signal change on T2-weighted MRI. The transverse ligament was observed inferior to the ossicle and superior to the hypoplastic dens. The cervicomedullary compression did not change on flexion-extension MRI. At the time of his ventral transoral-transpalatopharyngeal approach to the CVJ, the transverse ligament was seen below the anterior arch of the atlas and above the hypoplastic dens. The transverse ligament had slid inferior and anterior to the ossicle and was seen soon after exposure of the longus colli and longus capitis muscles. The $\mathrm{C}-1$ anterior tubercle and $\mathrm{C}-1$ anterior arch were removed to gain access to the dystopic OO, which had to be pulled above and anterior to the transverse ligament. With this there was good decompression of the tectorial membrane. A dorsal Oc-C3 fusion was made. Postoperative MRI showed satisfactory reduction with relief of the ventral cervicomedullary compression. He did well from a neurological standpoint and has returned to his previous activities.

\section{Case 3}

This 21-year-old woman presented with severe occipi- 


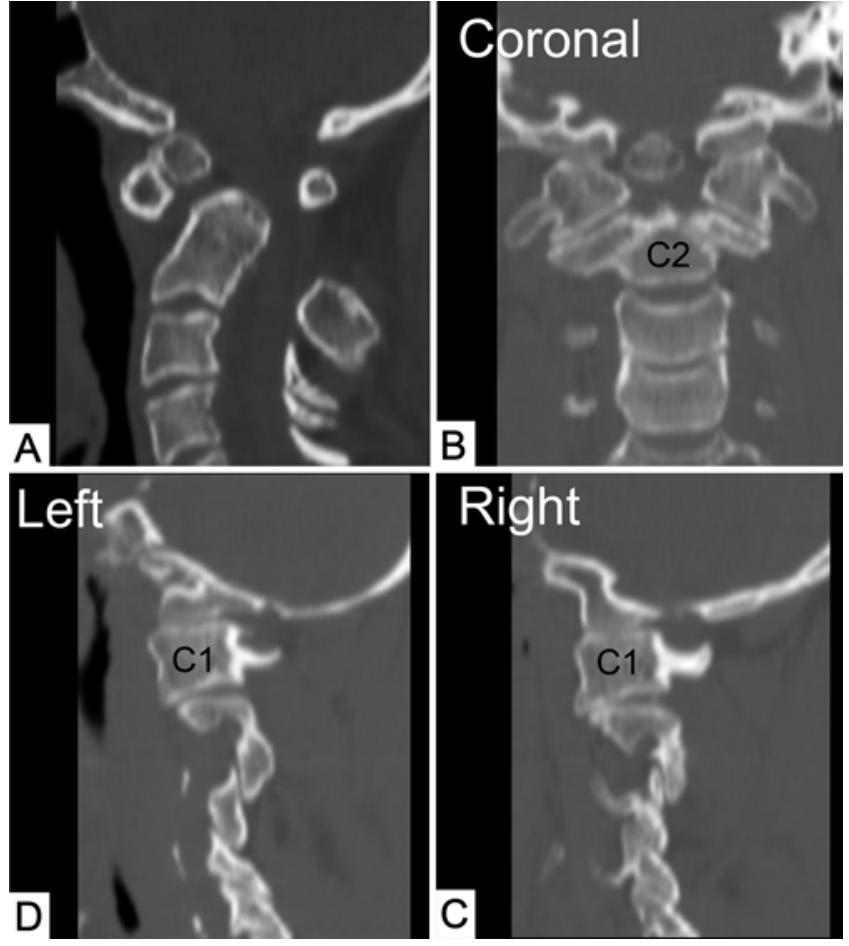

FIG. 2. Case 1. Facets of $\mathrm{C}-1$ and $\mathrm{C}-2$ are dislocated in irreducible $\mathrm{OO}$ but do not have osseous abnormalities that would prevent reduction. Preoperative CT imaging of irreducible $\mathrm{OO}$ in the sagittal (A) and coronal (B) planes and on the left (C) and right (D), illustrating C1-2 facet dislocation but no osseous abnormalities that would prevent reduction.

tal headaches of 6 months' duration, which were aggravated by head movement. She had episodic loss of function of the left arm and left leg. On examination she was unsteady in her gait and had a nearly absent gag response. There was gross hyperreflexia of the deep tendon reflexes in the upper and lower extremities.

The CT imaging of the CVJ identified a dystopic OO, which was attached to the lower clivus (Fig. 4). The MRI studies revealed ventral mid-medulla compression by the $\mathrm{OO}$, which resided behind the superior portion of the axis body. The transverse ligament was visualized lying over the hypoplastic OP and inferior and anterior to the ossicle. There was cerebellar tonsillar ectopia, with the tonsils extending below the posterior arch of $\mathrm{C}-1$ and the cervicomedullary buckle identified between the $\mathrm{C}-1$ and $\mathrm{C}-2$ vertebral body level. The patient was placed in crown halo cervical traction and an anterior transoral resection of the ossicle and superior aspect of the axis body was performed. It was confirmed that the ossicle was posterior to the transverse ligament. Postoperative MRI showed that the ventral medulla was decompressed and the cerebellar tonsils had ascended. Because of instability the patient underwent a dorsal Oc-C3 instrumentation with rib graft fusion. She made a complete neurological recovery.

\section{Discussion}

\section{Key Results and Interpretation}

In the largest series to date of irreducible $\mathrm{OO}$ and the only study to examine variable factors that lead to irreducibility in this entity, we found that the position of the transverse ligament anterior and inferior to the $\mathrm{OO}$ was present in all 41 cases. The ossicle was invariably positioned posterior to the transverse ligament and could not be manipulated forward intraoperatively to achieve reduction. No other variable examined was present in all cases, suggesting that the position of the transverse ligament was the defining factor in irreducibility. No osseous abnormalities of the atlantoaxial facets were found that would have prevented reduction. Given its presence in the majority of cases, granulation tissue also was likely to have contributed to preventing reduction, and probably formed after the ossicle slipped posterior to the transverse ligament. The dystopic variant of $\mathrm{OO}$ was also observed in the majority of cases, suggesting a predisposing factor to instability and irreducibility. Associated factors such as Down syndrome and segmentation failure of the subaxial spine occurred only in the pediatric population with irreducible $\mathrm{OO}$, suggesting that these factors may predispose patients to earlier presentation of instability and irreducibility. All of these key results are discussed below.

\section{Pathogenesis of 00 Leading to Instability and Irreducibility}

The origin of $\mathrm{OO}$ has been extensively debated in the literature. ${ }^{2}$ Some have suggested $\mathrm{OO}$ is a congenital and/ or developmental abnormality, which occurs after failure of the center of ossification of the dens to fuse with the body of C-2. This is supported by familial and identical twin cases of $\mathrm{OO}, 1,9,16,24$ suggesting a genetic component associated with faulty embryogenesis. Others have proposed that $\mathrm{OO}$ may represent an unremembered and/or unrecognized fracture through the $\mathrm{C}-2 /$ dens growth plate in childhood (nonhealed odontoid synchondrosis fracture) leading to a chronic nonunited fracture of the OP. The posttraumatic hypothesis is supported by case reports of patients with a previously documented intact C-2 who later were found to have $\mathrm{OO}$ after remote trauma.,12,21,23 It is likely that $\mathrm{OO}$ occurs more commonly from posttraumatic origins, with some cases being congenital.12,18 Additionally, there may also be cases that occur via both mechanisms.

Regardless of the origin of $\mathrm{OO}$ and of more clinical importance, $\mathrm{OO}$ can lead to $\mathrm{C} 1-2$ instability. $3,10,12,17,19,25$ The stability of the atlantoaxial articulation depends on the osseous integrity of the OP and associated C1-2 ligaments. The transverse ligament is a tight band positioned to trap the OP against the anterior arch of $\mathrm{C}-1$, thereby allowing physiological rotation of $\mathrm{C}-1$ on $\mathrm{C}-2$ and, at the same time, providing stability by preventing translational motion of $\mathrm{C}-1$ on $\mathrm{C}-2$ that could cause spinal cord injury. The transverse ligament represents the primary restraint to translational motion of $\mathrm{C}-1$ with respect to $\mathrm{C}-2$ because the atlantoaxial joint has flat lateral mass articulations, no intervertebral disc to limit motion, loose capsular ligaments, weak posterior ligaments, and lack of the ligamentum flavum. With OO, the lack of continuity of the ossicle with the body of C-2 hinders the ability of the transverse ligament to limit translational movement of $\mathrm{C}-1$ on the 

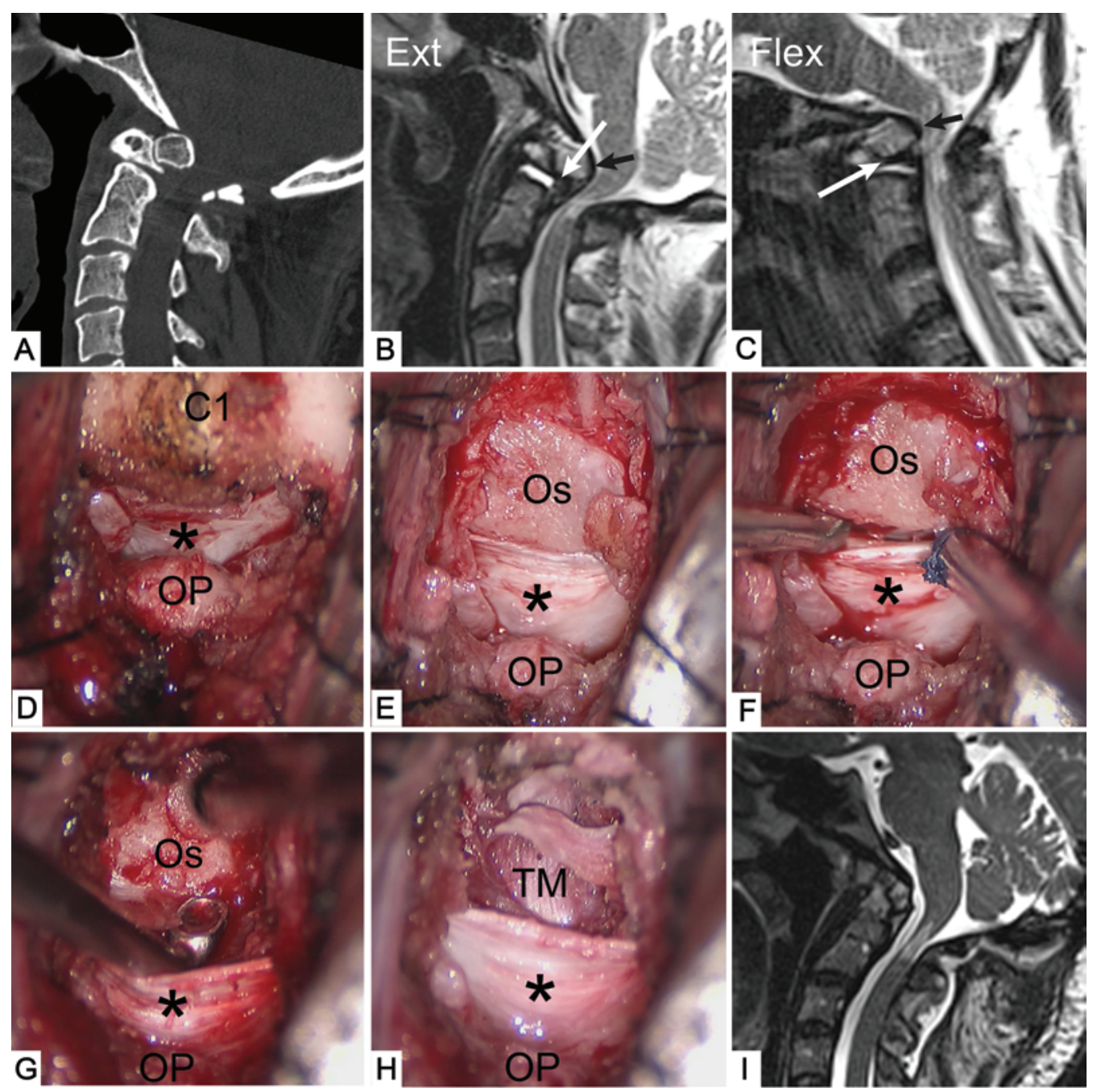

FIG. 3. Case 2. Intraoperative photographs and radiographic correlates of the transverse ligament positioned anterior and inferior to an $\mathrm{OO}$, thus preventing reduction and causing severe cervicomedullary compression in a 27 -year-old man. Preoperative sagittal $\mathrm{CT}$ imaging $(\mathbf{A})$ revealing a dystopic $\mathrm{OO}$. Extension $(\mathbf{B})$ and flexion $(\mathbf{C})$ sagittal T2-weighted MRI revealing irreducible $\mathrm{OO}$ and severe cervicomedullary compression and T2 cord signal change. The transverse ligament (white arrows) is hypointense with an associated hyperintense signal, probably from edematous changes. The transverse ligament is anterior and inferior to the ossicle, thus preventing reduction of the ossicle and therefore the ability to achieve normal alignment. The tectorial membrane (black arrows) lies in its normal position. Transoral approach and exposure of the C-1 anterior tubercle, C-1 anterior arch, and hypoplastic $\mathrm{OP}(\mathrm{D})$. The pathological position of the transverse ligament $\left(^{*}\right)$ - anterior and inferior to the ossicle — can be partly visualized. After removal of the $\mathrm{C}-1$ anterior tubercle $(\mathrm{E}-\mathbf{G})$, the ossicle is exposed and the transverse ligament can be clearly seen anterior and inferior to the ossicle. The ossicle is resected posterior and superior to the transverse ligament by using a combination of curettes, rongeurs, and electric drill $(\mathrm{H})$. This completely decompresses the CMJ. The tectorial membrane (TM) can then be visualized. The transverse ligament remains projecting superiorly and anteriorly over the hypoplastic OP. A postoperative sagittal T2-weighted MRI sequence (I) obtained after an Oc-C3 posterior fusion reveals complete decompression at the CMJ and proper CVJ alignment. Figure is available in color online only.

body of C-2. Thus, this can lead to instability whereby $\mathrm{C}-1$ and the ossicle can be free to move on C-2. Continued stress on the C1-2 joint, either with normal physiological movements of the neck, abrupt trauma, physical stress, or a predisposition to ligamentous instability can lead to incompetence of the transverse ligament and gross radiographic instability. ${ }^{12,22,26,27}$

\section{Stability of the Atlantoaxial Joint and the Transverse Ligament in Irreducible 00}

The initial instability of $\mathrm{OO}$ is often reducible with neck positioning, and normal anatomical alignment can be achieved. However, with repeated subluxations of $\mathrm{C}-1$ on $\mathrm{C}-2$, our findings suggest the incompetence of the transverse ligament reaches a point of irreversibility and slides inferior and slightly anterior to the OO. This position of the transverse ligament locks $\mathrm{C}-1$ and $\mathrm{C}-2$ into dislocation and prevents reduction of $\mathrm{C} 1-2$. Biomechanically, the position of the transverse ligament anterior and inferior to the ossicle creates a fixed irreducible position of the ossicle and an inability for reduction and realignment of the CVJ (Figs. 1-4). We suspect that because of this lack of move- 

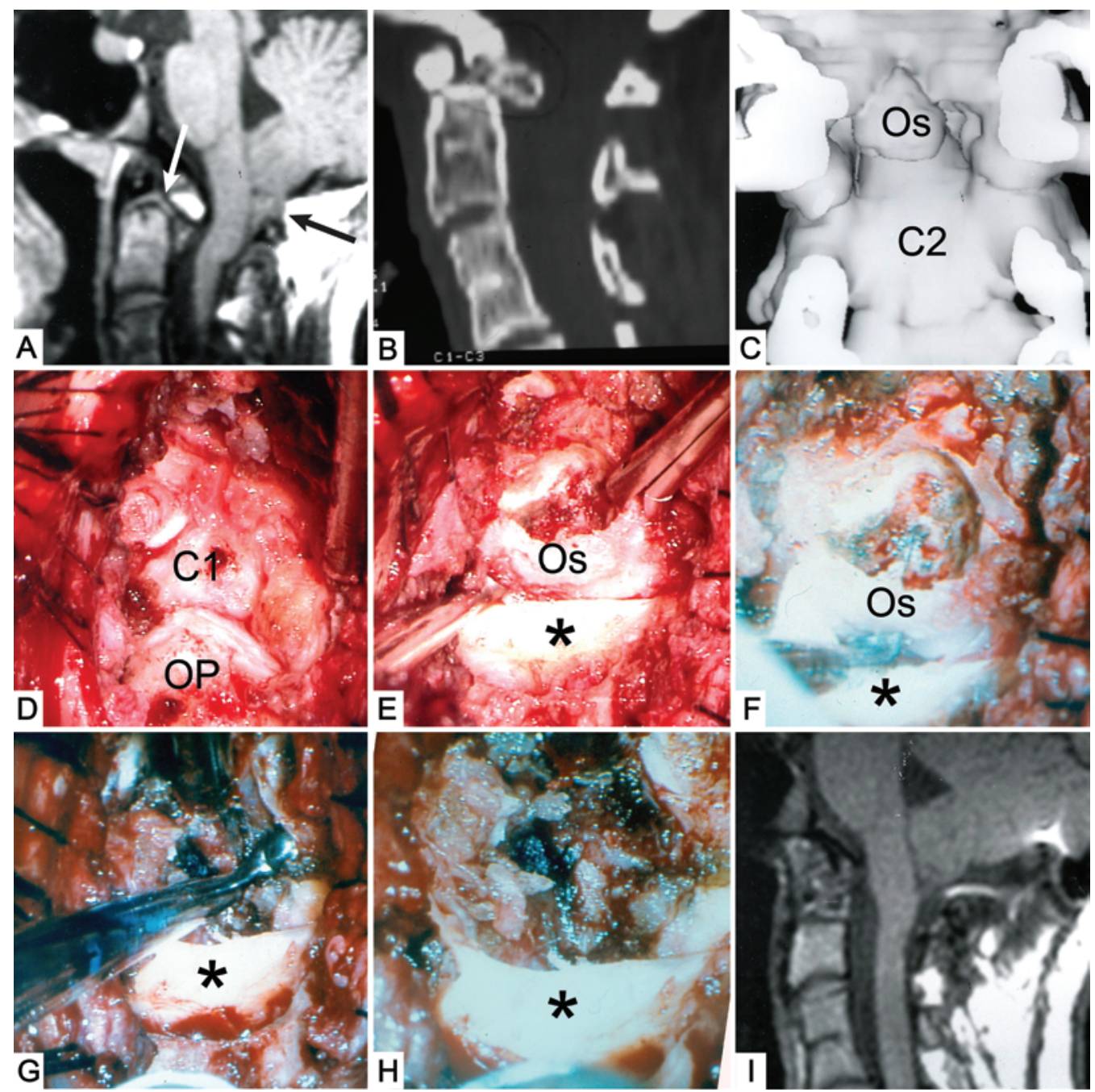

FIG. 4. Case 3. Dystopic 00 with pathological position of the transverse ligament causing irreducibility-this resulted in cervicomedullary compression, posterior displacement of the medulla, and Chiari I malformation. Sagittal T1-weighted MRI (A) revealing irreducible dystopic $\mathrm{OO}$ resulting in cervicomedullary compression, posterior displacement of the medulla, and Chiari I malformation (black arrow) with inferior displacement of the cervicomedullary buckle. The transverse ligament (white arrow) is partially hypointense, probably from edematous changes. The transverse ligament is anterior and inferior to the ossicle, thus preventing reduction. Preoperative sagittal (B) and coronal (C) CT imaging revealing a dystopic 00. Transoral approach and exposure of the C-1 anterior tubercle, C-1 anterior arch, and hypoplastic OP (D). The C-1 anterior tubercle, C-1 anterior arch, hypoplastic OP, and axis body is resected by decompressing the medulla and cervical spinal cord and exposing the $\mathrm{OO}$ (E and F). The pathological position of the transverse ligament $\left({ }^{*}\right)$-anterior and inferior to the ossicle —can now be visualized. The ossicle is resected posterior and superior to the transverse ligament by using a combination of curettes, rongeurs, and electric drill ( $\mathbf{G}$ and $\mathbf{H})$. This completely decompresses the CMJ. The transverse ligament remains projecting superiorly and anteriorly over the hypoplastic OP. A postoperative sagittal T1-weighted MRI study (I) obtained after an Oc-C3 posterior fusion reveals complete decompression at the $\mathrm{CMJ}$, normal brainstem position, resolution of the Chiari I malformation with ascent of the tonsils above the foramen magnum, and proper CVJ alignment. Figure is available in color online only.

ment due to irreducibility, granulation tissue around the ossicle forms over time.

\section{Imaging of the Transverse Ligament in 00}

The transverse ligament is visualized as a hypointense ligament on MRI, located just posterior to the OP and connected to the anterior arch on both sides of the dens. ${ }^{4,11}$ As we have shown, the transverse ligament in irreducible $\mathrm{OO}$ is visualized as a hypointense ligament lying inferior to the ossicle on MRI. There is an associated hyperintensity to the ligament that is compressible, which we suspect is indicative of edematous changes to the ligament (Fig. 1). The pathological ligament appears thickened on MRI.

\section{Differences Between Pediatric and Adult Population}

There appears to be a difference between pediatric and adult cases of irreducible OO. Individuals presenting in the pediatric age range were much more likely to have segmentation failure or Down syndrome, suggesting that these factors may influence the instability of the $\mathrm{OO}$ and affect the ligaments associated with the CVJ, predisposing to irreducible $\mathrm{OO}$ at earlier ages. Down syndrome re- 
sults in ligamentous laxity and predisposes to $\mathrm{Oc}-\mathrm{C} 1$ and $\mathrm{C} 1-2$ instability. ${ }^{20}$ Therefore, it's likely that the already lax transverse ligament in cases of Down syndrome and $\mathrm{OO}$ is more susceptible to ultimately being fixed anterior to the ossicle in OO. This incompetence may allow the ossicle to slide posterior to the transverse ligament and become fixed in that position, preventing reduction. Similar to Down syndrome, segmentation failure of the subaxial spine is also associated with instability at $\mathrm{C} 1-2$ because it places more stress on the $\mathrm{C} 1-2$ joint for normal physiological movements, predisposing to $\mathrm{C} 1-2$ instability and then irreducibility.

\section{Dystopic and Orthotopic 00}

Os odontoideum has been categorized into 2 anatomi-

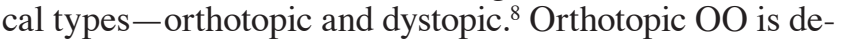
fined as an ossicle lying in the normal position of the typical OP and that moves with C-1 or in conjunction with the body of C-2. Dystopic OO is defined as an ossicle that is located near the occipital bone and that may be physically or functionally fused with the basion of the clivus, and that therefore moves separately from the body of C-2. Because the majority of patients in this case series of irreducible OO had the dystopic type, it's possible that a dystopic OO is more susceptible to instability and then irreducibility. We can hypothesize that a fixed ossicle makes it easier for the transverse ligament to slide inferior and then anterior to it.

\section{Limitations of the Study}

As with all retrospective studies, there is an inherent bias compared with prospective studies. However, this has been an ongoing study and assessment since 1978. We are limited in our analysis because we do not have a control group. We have not done a transoral approach for stable or reducible $\mathrm{OO}$ to compare intraoperative findings regarding the position of the transverse ligament. It is very possible that the position of the transverse ligament may be inferior to the ossicle in some unstable but reducible cases of OO. Our review of these reducible cases has not shown that it is. However, it was obvious from our intraoperative approach that the ossicle was physically incapable of sliding in front of the transverse ligament in the irreducible OO cases assessed in this study.

\section{Conclusions}

In the largest series to date of irreducible $\mathrm{OO}$ and the only study to examine variable factors that lead to irreducibility in this entity, we found that the position of the transverse ligament anterior and inferior to the ossicle is the most common factor in the irreducibility of OO. The presence of granulation tissue and an $\mathrm{OO}$ of the dystopic variant is also associated with irreducibility. The presence of Down syndrome and segmentation failure probably leads to faster progression of ligamentous incompetence and therefore earlier presentation of instability and irreducibility. This is the first study in which intraoperative findings regarding the transverse ligament have been correlated with MRI.

\section{References}

1. Al Kaissi A, Chehida FB, Ghachem MB, Grill F, Klaushofer $\mathrm{K}$ : Atlanto-axial segmentation defects and os odontoideum in two male siblings with opsismodysplasia. Skeletal Radiol 38:293-296, 2009

2. Arvin B, Fournier-Gosselin MP, Fehlings MG: Os odontoideum: etiology and surgical management. Neurosurgery 66 (3 Suppl):22-31, 2010

3. Dai L, Yuan W, Ni B, Jia L: Os odontoideum: etiology, diagnosis, and management. Surg Neurol 53:106-109, 2000

4. Debernardi A, D'Aliberti G, Talamonti G, Villa F, Piparo M, Collice M: The craniovertebral junction area and the role of the ligaments and membranes. Neurosurgery 68:291-301, 2011

5. Dlouhy BJ, Dahdaleh NS, Menezes AH: Evolution of transoral approaches, endoscopic endonasal approaches, and reduction strategies for treatment of craniovertebral junction pathology: a treatment algorithm update. Neurosurg Focus 38(4):E8, 2015

6. Dyck P: Os odontoideum in children: neurological manifestations and surgical management. Neurosurgery 2:93-99, 1978

7. Fielding JW, Griffin PP: Os odontoideum: an acquired lesion. J Bone Joint Surg Am 56:187-190, 1974

8. Fielding JW, Hensinger RN, Hawkins RJ: Os Odontoideum. J Bone Joint Surg Am 62:376-383, 1980

9. Flemming C, Hodson CJ: Os odontoideum; a congenital abnormality of the axis; case report. J Bone Joint Surg Br 37-B:622-623, 1955

10. Klimo P Jr, Kan P, Rao G, Apfelbaum R, Brockmeyer D: Os odontoideum: presentation, diagnosis, and treatment in a series of 78 patients. J Neurosurg Spine 9:332-342, 2008

11. Krakenes J, Kaale BR, Rorvik J, Gilhus NE: MRI assessment of normal ligamentous structures in the craniovertebral junction. Neuroradiology 43:1089-1097, 2001

12. Menezes AH: Pathogenesis, dynamics, and management of os odontoideum. Neurosurg Focus 6(6):e2, 1999

13. Menezes AH, Graf CJ, Hibri N: Abnormalities of the craniovertebral junction with cervico-medullary compression. A rational approach to surgical treatment in children. Childs Brain 7:15-30, 1980

14. Menezes AH, VanGilder JC: Transoral-transpharyngeal approach to the anterior craniocervical junction. Ten-year experience with 72 patients. J Neurosurg 69:895-903, 1988

15. Menezes AH, VanGilder JC, Graf CJ, McDonnell DE: Craniocervical abnormalities. A comprehensive surgical approach. J Neurosurg 53:444-455, 1980

16. Morgan MK, Onofrio BM, Bender CE: Familial os odontoideum. Case report. J Neurosurg 70:636-639, 1989

17. Rozzelle CJ, Aarabi B, Dhall SS, Gelb DE, Hurlbert RJ, Ryken TC, et al: Os odontoideum. Neurosurgery 72 (Suppl 2):159-169, 2013

18. Sankar WN, Wills BP, Dormans JP, Drummond DS: Os odontoideum revisited: the case for a multifactorial etiology. Spine (Phila Pa 1976) 31:979-984, 2006

19. Shirasaki N, Okada K, Oka S, Hosono N, Yonenobu K, Ono $\mathrm{K}$ : Os odontoideum with posterior atlantoaxial instability. Spine (Phila Pa 1976) 16:706-715, 1991

20. Taggard DA, Menezes AH, Ryken TC: Instability of the craniovertebral junction and treatment outcomes in patients with Down's syndrome. Neurosurg Focus 6(6):e3, 1999

21. Verska JM, Anderson PA: Os odontoideum. A case report of one identical twin. Spine (Phila Pa 1976) 22:706-709, 1997

22. Visocchi M, Di Rocco C: Os odontoideum syndrome: pathogenesis, clinical patterns and indication for surgical strategies in childhood. Adv Tech Stand Neurosurg 40:273-293, 2014

23. Wang S, Wang C: Acquired os odontoideum: a case report and literature review. Childs Nerv Syst 28:315-319, 2012 
24. Wang S, Wang C: Familial dystopic os odontoideum: a report of three cases. J Bone Joint Surg Am 93:e44, 2011

25. Watanabe M, Toyama Y, Fujimura Y: Atlantoaxial instability in os odontoideum with myelopathy. Spine (Phila Pa 1976) 21:1435-1439, 1996

26. Zhang Z, Wang H, Liu C: Acute traumatic cervical cord injury in pediatric patients with os odontoideum: a series of 6 patients. World Neurosurg 83:1180.e1-1880.e6, 2015

27. Zhao D, Wang S, Passias PG, Wang C: Craniocervical instability in the setting of os odontoideum: assessment of cause, presentation, and surgical outcomes in a series of 279 cases. Neurosurgery 76:514-521, 2015

\section{Disclosures}

The authors report no conflict of interest concerning the materials or methods used in this study or the findings specified in this paper.

\section{Author Contributions}

Conception and design: Dlouhy, Menezes. Acquisition of data: Dlouhy, Menezes. Analysis and interpretation of data: all authors. Drafting the article: Dlouhy. Critically revising the article: all authors. Reviewed submitted version of manuscript: all authors. Approved the final version of the manuscript on behalf of all authors: Dlouhy. Statistical analysis: Dlouhy. Study supervision: Menezes.

\section{Supplemental Information \\ Videos}

Video 1. https://vimeo.com/198207384.

\section{Correspondence}

Brian J. Dlouhy, Department of Neurosurgery, University of Iowa Children's Hospital, University of Iowa Hospitals and Clinics, 200 Hawkins Dr., Iowa City, IA 52242. email: brian-dlouhy@ uiowa.edu. 